\title{
NĂNG LỰC GIẢI QUYÊT VẤN ĐỀ TRONG CÁC TƯƠNG TÁC XÃ HộI \\ CỦA SINH VIÊN TRƯỜnG CAO ĐẲNG SU’ PHẠM QUẢNG TR!
}

\author{
The capacities of problem solving in social interaction \\ of students in Quang Tri teacher training college school
}

Ngày nhận bài: 21/01/2017; ngày phản biện: 22/2/2017; ngày duyệt đăng: 22/3/2017

Trần Thị Thanh Huyền*

\section{TÓM TẮT}

Năng lực giải quyết vấn đề trong các tương tác xã hội có vai trò đặc biệt quan trọng, nó giúp con người giải quyết tốt các tình huống xảy ra đối với bản thân, giữ thăng bằng tâm lí, thiết lập mối quan hệ xã hội và có thể thích ứng trong môi trường mới lạ. Trong khuôn khổ của bài báo này chúng tôi chỉ tập trung nghiên cứu năng lực duy trì, thiết lập các quan hệ xã hội và năng lực giải quyết vấn đề trong các tương tác xã hội của sinh viên sư phạm. Kết quả nghiên cứu cho thấy hai năng lực trên của sinh viên trường cao đẳng sư phạm Quảng Trị còn thấp, đặc biệt là năng lực thiết lập, duy trì các quan hệ xã hội. Phải chăng điểm trung bình cao hay thấp cũng chính là điểm mạnh, điểm yếu của sinh viên về các năng lực giải quyết vấn đề trong các tương tác xã hội. Trên cơ sở đó, chúng tôi đề xuất một số biện pháp nhằm nâng cao các năng lực trên cho sinh viên trường CĐSP Quảng Trị.

Từ khóa: Năng lục; giải quyết vấn đề; tuoong tác xã hộ; thiết lập quan hệ xã hộ; duy trì quan hệxã họi

\section{ABSTRACT}

The capacities of problem solving in social interaction have particularly important roles, it helps people solves situations which happen to yourself, keep balance mentality, set of social relationships and can adapt in the new environment. In the scope of this article, we only focus on the ability to maintain, establish social relationships and capacities of problem solving in social interaction of students in pedagogical college. The study results showed that two abilities of the students in of Quang Tri teacher training college is also low, particularly the capability set, maintain social ties. Reasonably, high or low average score of study results is also strong or weaknesses points of the students of capacities of problem solving in social interaction. On that basis, we propose a number of methods to enhance the capacity of problem solving in students of Quang Tri teacher training college school.

Key words: capacity; problem-solving; social interaction; establish social relationships; maintaining social relationships.

\section{1. Đặt vấn đề}

Năng lực giải quyết vấn đề (GQVĐ) trong các tương tác xã hội có vai trò đặc biệt quan trọng với không chỉ riêng một ai mà đối với tất cả mọi người. Bởi để tồn tại và phát triển con người ta phải tiếp xúc, quan hệ qua lại với rất nhiều cá nhân hay nhóm người để trao đổi về các vấn đề của công việc và cuộc sống. Vì vậy, năng lực giải quyết vấn đề giúp con người giải quyết tốt các tình huống xảy ra đối với bản thân, giữ thăng bằng tâm lí, thiết lập mối quan hệ xã hội và có thể thích ứng trong môi trường mới lạ.

Trong bối cảnh toàn cầu hóa và kinh tế

*Cao đẳng Sư phạm Quảng Trị 
tri thức được xem là thời cơ nhưng cũng là thách thức rất khốc liệt không chỉ đối với quốc gia mà còn đối với mỗi cá nhân con người. Vì vậy, để tồn tại và thích ứng tốt trong một môi trường xã hội luôn luôn biến đổi và phát triển không ngừng đòi hỏi mỗi người phải có năng lực thật sự để hội nhập vào cuộc sống mới với những yêu cầu mới. Trong xu thế đó, con người buộc phải nhanh chân trong cuộc chiến giành lấy tri thức, kỹ năng bằng cách học tập thường xuyên, học tập suốt đời để trụ vững trong vòng xoáy cuộc sống. Những ai không biết định hướng cuộc sống, không biết tự thân vận động chắc chắn sẽ bị bỏ lại phía sau, thậm chí bị đào thải bởi quy luật của kinh tế thị trường. Chính điều đó thúc đẩy con người phải không ngừng hoạt động sáng tạo, phát triển trình độ chuyên môn, kỹ năng, kỹ xảo và phải năng động để giải quyết tốt những vấn đề mà cuộc sống đặt ra cho bản thân mình. Vì vậy, việc hình thành năng lực GQVĐ cho người học là một vấn đề quan trọng và cấp bách nhất của dạy học và giáo dục.

Thực tế hiện nay cho thấy, giáo dục nước ta vẫn chưa chú trọng nhiều đến việc dạy cho người học có năng lực, đặc biệt là năng lực GQVĐ trong các tương tác xã hội. Vì thế, sinh viên ra trường chưa đáp ứng được yêu cầu của xã hội, yếu về thực hành chuyên môn, không có kỹ năng mềm, không thể giải quyết được những vấn đề của công việc cũng như của cuộc sống đặt ra. Chính vì thế mà giáo dục chưa tập trung vào việc dạy cho người học năng lực GQVĐ, khiến sinh viên ra trường chưa đáp ứng được yêu cầu của xã hội.

\section{Tổng quan nghiên cứu lý thuyết}

\subsection{Một số khái niệm liên quan}

\subsubsection{Khái niệm năng lụcc}

Phạm trù năng lực thường được hiểu theo những cách khác nhau và mỗi cách hiểu có những thuật ngữ tương ứng.
Năng lực (Capacity/Ability): Hiểu theo nghĩa chung nhất là khả năng (hoặc tiềm năng) mà cá nhân thể hiện khi tham gia một hoạt động nào đó ở một thời điểm nhất định.

Năng lực (Competence): Thường gọi là năng lực hành động: là khả năng thực hiện hiệu quả một nhiệm vụ/một hành động cụ thể, liên quan đến một lĩnh vực nhất định dựa trên cơ sở hiểu biết, kỹ năng, kỹ xảo và sự sẵn sàng hành động.

Năng lực được xây dựng dựa trên cơ sở tri thức, thiết lập qua giá trị, cấu trúc như là các khả năng, hình thành qua trải nghiệm/củng cố qua kinh nghiệm, hiện thực hóa qua ý chí (John Erpenbeck, 1998).

Năng lực: Là các khả năng và kỹ năng nhận thức vốn có ở cá nhân hay có thể học được... để giải quyết các vấn đề đặt ra trong cuộc sống. Năng lực cũng hàm chứa trong nó tính sẵn sàng hành động, động cơ, ý chí và trách nhiệm xã hội để có thể sử dụng một cách thành công và có trách nhiệm các giải pháp... trong những tình huống thay đổi (Weinert, 2001).

Năng lực: Là khả năng cá nhân đáp ứng các yêu cầu phức hợp và thực hiện thành công nhiệm vụ trong một bối cảnh cụ thể (OECD, 2002).

Năng lực: Là khả năng hành động, đạt được thành công và chứng minh sự tiến bộ nhờ vào khả năng huy động và sử dụng hiệu quả nhiều nguồn tích hợp của cá nhân khi giải quyết các vấn đề của cuộc sống. (Tremblay, 2002).

Năng lực: Là khả năng vận dụng kiến thức, kinh nghiệm, kỹ năng, thái độ và hứng thú để hành động một cách phù hợp và có hiệu quả trong các tình huống đa dạng của cuộc sống (Québec - Ministere de l'Education, 2004).

Năng lực là khả năng ứng phó thành công hay năng lực thực hiện hiệu quả một 
loại/lĩnh vực hoạt động nào đó trên cơ sở hiểu biết (tri thức), biết cách lựa chọn và vận dụng tri thức, kinh nghiệm, kỹ năng/kỹ xảo... để hành động phù hợp với những những mục tiêu và điều kiện thực tế hay hoàn cảnh thay đổi.

Điểm chung của các khái niệm năng lực:

Năng lực là khả năng hoàn thành nhiệm vụ đặt ra, gắn với một loại hoạt động nào đó. Năng lực là một yếu tố của nhân cách nên mang dấu ấn cá nhân, thể hiện tính chủ quan trong hành động, được hình thành theo quy luật hình thành và phát triển của nhân cách, trong đó giáo dục, hoạt động, giao lưu có vai trò quyết định. Mặt khác, về bản chất, năng lực được tạo nên bởi các thành tố: kiến thức, kỹ năng, kỹ xảo, các yếu tố này không tồn tại riêng lẻ mà chúng hòa quyện, đan xen vào nhau. Do đó, năng lực ở mỗi con người có được nhờ sự bền bỉ, kiên trì học tập, hoạt động, rèn luyện và trải nghiệm của bản thân.

Theo Nguyễn Công Khanh (2013) đã đưa ra định nghĩa làm việc về năng lực như sau: Năng lực là khả năng làm chủ nhũng hệ thống kiến thức, kỹ năng, thái độ và vận hành (kết nối) chúng một cách hợp lý vào thực hiện thành công nhiệm vu hoạc giải quyết hiệu quả vấn đề đặt ra của cuộc sống. Năng lực là một cấu trúc động (trừu tượng), có tính mở, đa thành tố, đa tầng bậc, hàm chứa trong nó không chỉ là kiến thức, kỹ năng,... mà cả niềm tin, giá trị, trách nhiệm xã hội... thể hiện ở tính sẵn sàng hành động trong những điều kiện thực tế, hoàn cảnh thay đổi.

\subsubsection{Giải quyết vấn đề}

Giải quyết vấn đề là hoạt động làm cho ai đó đạt được mong muốn từ nền tảng ban đầu mà cách thức đạt đến mong muốn vẫn chưa rõ ràng tại thời điểm đó (Charness, 1998) .

Giải quyết vấn đề là quá trình tự định hướng nhận thức - hành vi mà một cá nhân hoặc nhóm cố gắng để xác định hoặc khám phá các giải pháp hiệu quả cho từng vấn đề cụ thể gặp phải trong cuộc sống. Đặc biệt, quá trình nhận thức - hành vi nghĩ ra nhiều khả năng, giải pháp hiệu quả cho từng vấn đề cụ thể và làm tăng khả năng lựa chọn các giải pháp hiệu quả nhất trong số các lựa chọn thay thế khác nhau (D'Zurilla \& Goldfried, 1997).

Giải quyết vấn đề là quá trình tự nhận thức, hành động trong đó cá nhân cố gắng xác định vấn đề và đưa ra các giải pháp phù hợp với từng vấn đề cụ thể trong cuộc sống hàng ngày (D'Zurilla, Nezu, 2001).

Giải quyết vấn đề là quá trình đạt đến mục tiêu mà cách thức tiến hành chưa rõ ràng (Martinez, 2005)

Giải quyết vấn đề là mục tiêu hướng suy nghĩ và hành động trong những tình huống mà thường là chưa có sẵn giải pháp. Giải quyết vấn đề ít nhiều đã có mục tiêu xác định, nhưng không phải ngay lập tức biết cách làm thế nào để đạt được nó. Sự không phù hợp về mục tiêu và các phương pháp giải quyết tạo thành vấn đề. Hiểu các giải pháp và từng bước xây dựng kế hoạch, tìm hiểu nguyên nhân, thu nhận thông tin tạo thành quá trình giải quyết vấn đề (Jean-Paul Reeff, Anouk Zabal,Christine Blech,2006).

Giải quyết vấn đề là khả năng của một cá nhân sử dụng quá trình nhận thức để đương đầu và giải quyết các tình huống thực tiê̂n trong lúc phương pháp và cách thức hành động chưa rõ ràng và không thuộc một lĩnh vực nhất định (K.Carroll \&B.Chettri).

\subsubsection{Tương tác xã hội}

Tương tác xã hội là những hành vi, hành động, hoặc hoạt động qua lại giữa hai hay nhiều người định hướng bản thân lẫn nhau, nghĩa là, bất kì những hành vi nào cố gắng gây ảnh hưởng hay tạo ra sự đánh giá lẫn nhau về kinh nghiệm chủ quan hoặc ý định của họ . Điều này có nghĩa là các bên tham gia tương tác xã hội 
phải biết lẫn nhau - để lại trong tâm trí của mỗi người. (R.J. Rummel, 1976).

Theo từ điển tâm lý học, tương tác xã hội là quá trình tác động qua lại trực tiếp hoặc gián tiếp giữa các chủ thể xã hội, từ đó phát sinh ra các mối liên hệ, quan hệ xã hội gắn kết các con người với nhau. Tâm lý học nghiên cứu tương tác xã hội ở các phương diện: 1) Sự ảnh hưởng lẫn nhau, thể hiện ra trong các mối quan hệ, giao tiếp, cảm xúc, tình cảm chung; 2) Sự tác động lẫn nhau diễn ra trong quá trình thực hiện hoạt động cùng nhau, trong phân phối, tiêu dùng xã hội, tác động ảnh hưởng lẫn nhau; 3) Quá trình trao đổi các thông tin, các suy nghĩ, tư tưởng, các ý kiến, tác động ảnh hưởng lên nhận thức, nhu cầu, động cơ hoạt động của nhau. Tương tác xã hội có cấu trúc phức tạp, mang tính hệ thống, đóng vai trò là cái điều chỉnh, điều khiển mạnh mẽ hành vi hoạt động của các cá nhân.

Dưới góc độ xã hội học, tương tác xã hội là sự tác động qua lại giữa các chủ thể xã hội với nhau trong cộng đồng mà qua đó mỗi cá nhân có thể nhận diện chính bản thân mình đồng thời có thể nhận diện được người khác thông qua nhãn quan xã hội của họ.

Tương tác xã hội là hình thức giao tiếp xã hội hay sự trao đổi giữa các cá nhân và cộng đồng, trong mối quan hệ qua lại đó hành động xã hội được diễn ra để thích ứng của một hành động này với một hành động khác. Đồng thời qua đó cũng tìm thấy điểm chung trong sự hiểu biết tình huống, ý nghĩa hành động nhằm đạt được mức độ hợp tác nhất định hoặc sự đồng tình giữa chúng.

Trong nghiên cứu của mình, chúng tôi xác định khái niệm tương tác xã hội là quá trình tác động qua lại trực tiếp hoặc gián tiếp giữa các chủ thể xã hội, từ đó phát sinh ra các mối liên hệ, quan hệ xã hội gắn kết giữa các con người với nhau. Sự tương tác xã hội được xem xét ở trên phương diện là sự ảnh hưởng lẫn nhau qua các mối quan hệ, giao tiếp, cảm xúc, tình cảm chung.

Năng lực GQVĐ trong các tương tác xã hội là năng lực làm chủ, điều khiển, kiểm soát, quản lý có hiệu quả các tương tác xã hội thể hiện ở khả năng nhận biết, hiểu rõ bản chất vấn đề, phát hiện giải pháp, đánh giá từng giải pháp, chọn lựa giải pháp phù hợp nhất để giải quyết hiệu quả các vấn đề nảy sinh trong các hoạt động cùng/với người khác. Đó chính là khả năng ứng xử một cách thông minh trong những tình huống tương tác xã hội (liên nhân cách). Nó cho phép cá nhân sử dụng có hiệu quả các chiến lược giải quyết vấn đề để đạt được những mục đích trong các hoạt động tương tác xã hội (Nguyễn Công Khanh, 2011).

Như vậy, năng lực GQVĐ là một dạng năng lực thuộc trí tuệ xã hội thể hiện năng lực GQVĐ một cách thông minh trong các hoạt động giao tiếp, ứng xử/tương tác cùng/với người khác. Sống và hoạt động trong cộng đồng cùng/với người khác đòi hỏi mỗi cá nhân phải chú ý đến các quy luật xã hội, sự thừa nhận và đánh giá theo chuẩn mực xã hội. Điều này đòi hỏi mỗi cá nhân phải có năng lực nhận biết, đánh giá và chuẩn đoán phù hợp về hành động của người khác, để từ đó tổ chức, đặt kế hoạch và quyết định chọn lựa những hành động phù hợp cho mình để đạt hiệu quả tương tác, đạt mục đích, ngay cả khi gặp phải những điều kiện hoàn cảnh không thuận lợi.

\subsection{Thiết kế bộ công cụ đo năng lực} giải quyết vấn đề trong các tương tác xã hội

Trắc nghiệm đo năng lực GQVĐ trong các tương tác xã hội được xây dựng dựa trên quan niệm: Năng lực giải quyết vấn đề trong các tương tác xã hội là năng lực làm chủ, điều khiển, kiểm soát, quản lý có hiệu quả các tương tác xã hội thể hiện ở khả năng nhận biết, hiểu rõ bản chất vấn đề, phát hiện giải pháp, đánh giá 
từng giải pháp, chọn lựa giải pháp phù hợp nhất để giải quyết hiệu quả trong các tương tác xã hội đặc trưng của sinh viên sư phạm.

Từ định nghĩa này chúng tôi đề xuất mô hình cấu trúc năng lực GQVĐ trong các tương tác xã hội của sinh viên, sao cho dựa vào mô hình này có thể thiết lập được một phép đo theo cách tiếp cận năng lực (bộc lộ tối đa khả năng của cá nhân). Mô hình cấu trúc năng lực GQVĐ trong các tương tác xã hội gồm 2 thành tố sau:

\section{- Năng lực thiết lập, duy trì các quan hệ} $x a \tilde{h} h \hat{i} i$ : Khả năng thấu hiểu các quan hệ xã hội, biết cách thiết lập, duy trì và phát triển nó một cách hợp lý. Các năng lực này rất quan trọng trong việc tạo dựng các mối quan hệ tương tác bền vững, hiệu quả. Đây là một phức hợp năng lực hành động có tính toán, cân nhắc... để ứng dụng một chiến lược nuôi dưỡng phát triển các quan hệ tương tác tích cực.

- Năng lực giải quyết vấn đề trong các turong tác xã họi: Gồm các năng lực cho phép cá nhân xác định bản chất vấn đề, phát hiện giải pháp, đánh giá từng giải pháp, chọn lựa được giải pháp phù hợp nhất để giải quyết các vấn đề nảy sinh trong các hoạt động cùng/với người khác. Năng lực giải quyết vấn đề trong các tình huống tương tự trên đây chính là khả năng ứng xử một cách thông minh trong những tình huống tương tác liên nhân cách. Nó cho phép cá nhân sử dụng có hiệu quả các chiến lược giải quyết vấn đề để đạt được những mục đích trong các hoạt động tương tác xã hội.

Trắc nghiệm gồm 40 items được cấu trúc thành 2 thang đo tương ứng với 2 thành tố trên. Thang đo thứ nhất về năng lực duy trì, thiết lập quan hệ xã hội gồm 10 items, thang đo thứ hai về năng lực GQVĐ trong các tương tác xã hội gồm 30 items.
Mỗi item là một câu hỏi, một tình huống có vấn đề phải giải quyết. Mỗi tình huống này có 4 phương án lựa chọn, nghiệm thể (sinh viên) được yêu cầu cân nhắc, phát hiện các mối liên hệ trong kinh nghiệm, tri thức của cá nhân để đánh giá từng phương án nhằm tìm ra phương án thích hợp nhất. Bộ trắc nghiệm này được làm với cá nhân hoặc nhóm. Thời gian hoàn thành trắc nghiệm khoảng 30 phút.

\section{Cách tính điểm:}

Bởi vì đây là trắc nghiệm được thiết kế theo cách tiếp cận năng lực (bộc lộ tối đa khả năng của cá nhân) chứ không phải là tự đánh giá nên không thể sử dụng cách cho điểm nhiều phương án đúng (hay tất cả các phương án đều cho điểm). Ở đây chúng tôi chỉ cho điểm phương án đúng (1 điểm), còn các phương án nhiễu không cho điểm.

3. Kết quả nghiên cứu về năng lực gqvđ trong các tương tác xã hội của sinh viên trường cđ̛sp Quảng Trị

\subsection{Phương pháp tiếp cận nghiên cứu}

- Phương pháp nghiên cứu lý luận, hồi cứu tư liệu: nghiên cứu các tài liệu trong và ngoài nước liên quan đến năng lực giải quyết vấn đề trong các tương tác xã hội.

- Phương pháp trắc nghiệm: Xây dựng bộ trắc nghiệm đo lường năng lực GQVĐ trong các tương tác xã hội dựa trên 2 nhóm: năng lục duy trì các quan hệ xã hội và năng lục giải quyết vấn đề trong các tuoơng tác xã họi của sinh viên.

- Phương pháp chuyên gia: Tham khảo ý kiến của chuyên gia có kinh nghiệm trong lĩnh vực Đo lường đánh và đánh giá trong giáo dục về mô hình lý thuyết và xây dựng công cụ đo năng lực GQVĐ trong các tương tác xã hội dành cho sinh viên;

- Phương pháp thống kê toán học (sử dụng phần mềm SPSS và Quest để xử lý, phân tích số liệu). 


\subsection{Mẫu nghiên cứu}

Số lượng sinh viên của trường tuyển hàng năm không nhiều, tổng số sinh viên hiện đang theo học ở trường là 1.432 sinh viên, trong đó chúng tôi chỉ chọn điều tra trên 469 sinh viên. Số liệu điều tra chính thức về khách thể nghiên cứu được trình bày ở bảng sau:

\begin{tabular}{|c|l|c|}
\hline TT & \multicolumn{1}{|c|}{ Khoa/tổ } & Số lượng SV \\
\hline 1 & Tiểu học & 178 \\
\hline 2 & Mầm non & 91 \\
\hline 3 & CNTT & 44 \\
\hline 4 & Xã hội & 67 \\
\hline 5 & Tự nhiên & 69 \\
\hline 6 & Tâm lí - Anh văn & 20 \\
\hline & Cộng & $\mathbf{4 6 9}$ \\
\hline
\end{tabular}

3.3. Kết quả đánh giá thử nghiệm bộ công cụ

Chúng tôi đã dùng phương pháp đánh giá mức độ tương quan giữa các item trong cùng miền đo (consistency methods), sử dụng mô hình tương quan alpha của Cronbach (Cronbach's Cofficient Alpha). Mô hình này đánh giá độ tin cậy của phép đo dựa trên sự tính toán phương sai của từng item trong từng thang đo, toàn bộ phép đo và tính tương quan điểm của từng item với điểm của các item còn lại trên từng thang đo và của cả phép đo. Độ tin cậy của thang đo được coi là thấp nếu hệ số alpha $<0,40$. Kết quả đánh giá độ tin cậy cho thấy trắc nghiệm đo năng lực GQVĐ trong các tương tác xã hội có hệ số tin cậy Alpha ( $\mathrm{r}=$ $0,83)$ đạt mức khá cao. Điều này có nghĩa là bộ công cụ trắc nghiệm năng lực GQVĐ trong các tương tác xã hội đảm bảo yêu cầu về độ tin cậy.

Kết quả phân tích độ khó từng item của trắc nghiệm đo năng lực GQVĐ trong các tương tác xã hội theo mô hình Rasch cho thấy tất cả các item có độ khó tính theo thang logit phân bố rải đều từ tương đối dễ đến khó (từ 1.51 đến +2.0 ). Không có item nào quá dễ, có 2 item hơi khó. Tất cả các item của trắc nghiệm đo năng lực GQVĐ trong các tương tác xã hội đều nằm trong khoảng giữa hai đường chấm mờ, đáp ứng mô hình Rasch.

Kết quả đánh giá độ phân biệt của từng item cho thấy hầu hết các item có độ phân biệt nằm trong khoảng $0,2-0,4$ đáp ứng các tiêu chuẩn đo lường.

Một trắc nghiệm tốt, ngoài độ tin cậy tốt, cần phải có độ hiệu lực tốt. Chúng tôi đã sử dụng phương pháp phân tích nhân tố để đánh giá độ hiệu lực cấu trúc của trắc nghiệm. Kết quả phân tích nhân tố cho thấy trắc nghiệm đo năng lực GQVĐ chiết xuất được một nhân tố chung, có trị số đặc trưng (Intial Eigenvalues) là 6,281 (phương sai/biến thiên của các thành tố bộ phận). Nhân tố chung này giải thích cho $15,7 \%$ tổng biến thiên của của thang đo được đưa vào chiết xuất nhân tố. Phép thử KMO $(0,75)$ và phép thử Bartlett cũng khẳng định rằng có khả năng chiết xuất factor của một ma trận tương quan.

Kết quả đánh giá hệ số chứa (factor loadings - hệ số tương quan với nhân tố chung) cũng cho thấy hầu hết các item có tương quan khá chặt với các nhân tố chung. Điều này có thể chứng minh độ hiệu lực cấu trúc của phép đo năng lực GQVĐ trong các tương tác xã hội phù hợp với mô hình lý thuyết mà chúng tôi đã đề xuất.

Từ những kết quả phân tích trên đây cho thấy, trắc nghiệm đo năng lực GQVĐ trong các tương tác xã hội, về cơ bản đảm bảo các đặc tính thiết kế và các đặc tính đo lường. Nhìn chung hầu hết các item của trắc nghiệm đều đảm bảo có đủ độ tin cậy và độ hiệu lực. 
3.4. Năng lực GQVĐ của sinh viên trường CĐSP Quảng Trị

Kết quả phân tích đánh giá điểm trung bình (điểm thô) trên các tiểu thang đo và điểm tổng (điểm thô) của trắc nghiệm đo năng lực GQVĐ trong các tương tác xã hội của sinh viên được trình bày trong bảng sau:

\begin{tabular}{|l|c|c|}
\hline \multirow{2}{*}{$\begin{array}{c}\text { Năng lực GQVĐ trong } \\
\text { các tương tác xã hội }\end{array}$} & $\begin{array}{c}\text { Trung } \\
\text { bình }\end{array}$ & $\begin{array}{c}\text { Độ } \\
\text { lệch } \\
\text { chuẩn }\end{array}$ \\
\cline { 2 - 3 } & & 0,10 \\
\hline $\begin{array}{l}\text { Năng lực thiết lập, duy trì } \\
\text { các quan hệ xã hội }\end{array}$ & 5,47 & 0,26 \\
\hline $\begin{array}{l}\text { Năng lực GQVĐ trong các } \\
\text { tương tác xã hội }\end{array}$ & 12,72 & 0,31 \\
\hline $\begin{array}{l}\text { Năng lực GQVĐ trong các } \\
\text { tương tác xã hội } \\
\text { (Tổng điểm thô của trắc } \\
\text { nghiệm) }\end{array}$ & 18,18 & \\
\hline
\end{tabular}

Kết quả phân tích điểm trung bình (điểm thô) trên các tiểu thang đo của trắc nghiệm đo năng lực GQVĐ trong các tương tác xã hội cho thấy thang đo năng lực GQVĐ trong các tương tác xã hội điểm trung bình $=12,72$; thang đo năng lực thiết lập, duy trì các quan hệ xã hội điểm trung bình $=5,47$. Kết quả phân tích các item cho thấy các item đều phù hợp, cách tính điểm số của các item lại giống nhau (mỗi item trả lời đúng nhất - theo ý kiến của chuyên gia, sẽ đều được 1 điểm). Vậy phải chăng điểm trung bình cao hay thấp ở các tiểu thang đo cũng chính là những điểm mạnh/điểm yếu về các năng lực GQVĐ trong các tương tác xã hội tương ứng của sinh viên trường $\mathrm{CĐSP}$ Quảng Trị. Có thể nói rằng, sinh viên trường CĐSP Quảng Trị rất yếu về năng lực thiết lập, duy trì các quan hệ xã hội.

Theo các nhà tâm lí học, năng lực GQVĐ là năng lực thuộc trí tuệ xã hội. Đây một dạng trí tuệ mới, liên quan đến nhận thức xã hội và năng lực GQVĐ một cách thông minh trong các hoạt động giao tiếp, ứng xử/ tương tác cùng người khác.

Đặc biệt đối với sinh viên sư phạm - là những thầy cô giáo tương lai, đối tượng hoạt động nghề nghiệp đặc thù của sinh viên sư phạm là con người - học sinh/đồng nghiệp/phụ huynh học sinh. Môi trường hoạt động đặc trưng của sinh viên sư phạm là cùng và với người khác. Do vậy sinh viên CĐSP cần phải có khả năng nhạy cảm trong các tình huống giao tiếp, tương tác, thấu hiểu nhu cầu, hứng thú, tình cảm, hoàn cảnh của từng học sinh, có khả năng kiểm soát, hoá giải các xung đột, khuyến khích các tương tác nhóm... một phổ rộng lớn các năng lực nhận thức, năng lực hành động thuộc về phạm trù trí tuệ xã hội. Các ứng xử xã hội của sinh viên sư phạm trong các tình huống tương tác xã hội luôn đòi hỏi tính mô phạm - một yêu cầu về tính mẫu mực, hiệu quả, thể hiện đạo đức nghề nghiệp và luôn giàu cảm xúc tích cực.

\section{Kết luận}

Năng lực giải quyết vấn đề giúp con người giải quyết tốt các tình huống xảy ra đối với bản thân, giữ thăng bằng tâm lí, thiết lập mối quan hệ xã hội và có thể thích ứng trong môi trường mới lạ.

Đặc biệt đối với sinh viên sư phạm những thầy cô giáo trong tương lai, đối tượng hoạt động nghề nghiệp đặc thù của sinh viên sư phạm là con người - học sinh/đồng nghiệp/phụ huynh học sinh. Môi trường hoạt động đặc trưng của sinh viên sư phạm là cùng/ và với người khác. Chính vì vậy, năng lực GQVĐ trong các tương tác xã hội đối với sinh viên có vai trò vô cùng quan trọng. Thế nhưng kết quả điều tra khảo sát lại cho thấy sinh viên trường CĐSP Quảng Trị còn rất yếu về năng 
lực giải quyết vấn đề đặc biệt là năng lực thiết lập và duy trì các mối quan hệ xã hội.

Với những kết quả nghiên cứu đạt được, chúng tôi đề xuất một số biện pháp nhằm nâng cao năng lực GQVĐ trong các tương tác xã hội cho sinh viên trường CĐSP. Trường cần phải nhanh chóng đổi mới chương trình giáo dục, phương pháp giáo dục hướng đến phát triển các năng lực tương tác xã hội. Giáo dục cần đầu tư hơn cho công tác bồi dưỡng kỹ năng sống, đa dạng hóa các loại hình câu lạc bộ, tổ chức nhiều hoạt động ngoại khóa phù hợp, hấp dẫn thu hút mọi sinh viên tham gia. Qua đó, sinh viên được giáo dục giá trị sống, rèn luyện kỹ năng ứng xử sư phạm, giải quyết vấn đề, giao tiếp... nhằm tăng cơ hội thành công nghề nghiệp và cơ hội thành đạt của sinh viên sau khi ra trường.

\section{TÀI LIỆ THAM KHẢO}

1. A.G.Covaliov (1971), Tâm lí học cá nhân, Nxb Giáo dục, Hà Nội.

2. Edward C.Chang, Thomas J. D'Zurilla , Lawrence J.Sanna (2002), Social problem solving , American Psychological Association Washington, DC

3. Phạm Tất Dong (1977), Năng lực, Tạp chí Nghiên cưu Giáo dục, số 11

4. Nguyễn Công Khanh (2013), "Một số vấn đề về năng lực và xây dựng khung năng lực trong chương trình giáo dục phổ thông sau năm 2015", Báo cáo tại Hội thảo READ, Hà Nội, tr 250258.

5. Nguyễn Công Khanh - chủ nhiệm đề tài (2011), "Nghiên cứu chỉ số trí tuệ xã hội (SQ) của sinh viên các trường ĐHSP”, đề tài cấp Bộ, mã số B2009 -17 - 176.

6. Jean-Paul Reeff, Anouk Zabal,Christine Blech, 2006, The Assessment of Problem-Solving Competencies

7. Yunus et. al (2006), Problem Solving Abilities of Malaysian University Students, , International Journal of Teaching and Learning in Higher Education. 